\title{
INNOVACIÓN TECNOLÓGICA Y CRECIMIENTO REGIONAL EN MÉXICO, 1995-2000
}

\author{
Jorge Eduardo Mendoza* \\ Departamento de Estudios Económicos, El Colegio de la Frontera Norte \\ Víctor Hugo Torres \\ Facultad de Economía, Universidad de Colima
}

(Recibido 21 de mayo 2002, aceptado 5 de junio 2002)

\begin{abstract}
Resumen
Diversos estudios han encontrado evidencia de un proceso reciente de divergencia económica regional en México. En este estudio se aborda el problema de la desigualdad regional a partir del modelo neoclásico de convergencia. Así mismo, se analiza el efecto de la innovación tecnológica en el proceso de crecimiento económico regional en el periodo 1995-2000. Los resultados corroboran la existencia de un proceso de divergencia económica regional en dicho periodo. En este contexto, el efecto positivo de la innovación tecnológica no sólo favorece el crecimiento, también fomenta la divergencia regional. Esto se asocia al reciente desempeño de la actividad de innovación nacional, concentración regional, el grado de difusión tecnológica, así como con las políticas tecnológicas regionales.
\end{abstract}

\section{Abstract}

Diverse studies have found evidence of a recent process of regional economic divergence in Mexico. In this study, the problem of the regional inequality is approached from the neoclassic model of convergence. The effect of the technological innovation in the process of regional economic growth in period $1995-2000$ is analyzed, as well. The results confirm the existence of a process of regional economic divergence in this period. In such a context, the positive effect of the technological innovation not only favors growth, also promotes the regional divergence. This is associated to the recent performance of the activity of national innovation, the regional concentration of this activity, the degree of technological difussion, as well as the need to strengthen the regional technological policies.

Clasificación JEL: $\mathrm{O4}, \mathrm{O} 3$

Palabras Clave: Crecimiento económico, Tecnología regional e innovación tecnológica

* Coordinación de la Maestría en Economía Aplicada, Departamento de Economía, El Colegio de la Forntera Norte. Blyd. Abelardo L. Rodríguez, No. 2925 Zona Río C.P. 22320, Teléfono (01664)6326321, Tijuana, B.C. México Correo Electrónico emendoza@colef.mx

Los autores desean agradecer a dos dictaminadores anónimos sus valiosas observaciones. 


\section{Introducción}

El debate sobre el crecimiento económico se ha centrado fundamentalmente en el estudio de las causas del crecimiento. En esta discusión se encuentran los modelos de crecimiento endógeno, en los que se resalta por un lado la innovación tecnológica endógena como factor de crecimiento (Romer, 1990 y Jones, 1999) y, por otro, las extensiones del modelo neoclásico de Solow para estudiar el proceso de convergencia entre regiones y países (Barro y Sala-j-Martin, 1991).

En México, el análisis del crecimiento económico se ha enfocado recientemente al estudio de los determinantes del proceso de expansión económica. Esto ha permitido abordar el problema de la desigualdad económica regional y sus posibles implicaciones para el bienestar, ante el efecto de la política fiscal (Venegas-Martínez, 1999), del capital humano (Esquivel, 1999) y de las políticas de inversión pública en infraestructura económica y social (Fuentes y Mendoza, 2001).

El estudio de la convergencia/divergencia regional en México requiere incorporar el efecto de la innovación tecnológica, particularmente a partir de la crisis de 1994. Con base en la ecuación neoclásica de convergencia, este trabajo incluye la variable innovación tecnológica básica para estudiar la desigualdad regional en México. Los objetivos de la investigación son determinar si, a partir de la crisis de 1994, se ha generado un proceso de desigualdad económica regional en México y estimar el efecto de la innovación tecnológica en la desigualdad económica regional.

El presente estudio se divide en cuatro secciones. En la primera se expone la hipótesis de convergencia y se discuten algunos trabajos empíricos realizados para México. En la segunda sección, se realiza un análisis del producto interno bruto (PIB), así como de la innovación tecnológica por estados. En la tercera sección se presentan los resultados econométricos y en la última sección se presentan las conclusiones obtenidas.

\section{La hipótesis de convergencia en el crecimiento económico}

Uno de los resultados del modelo de crecimiento neoclásico propuesto por SolowSwan (1956) señala que las economías que poseen estructuras similares, y difieren en sus niveles iniciales de capital por persona $\left(k_{0}\right)$ y producto por persona $\left(y_{0}\right)$, convergerán a un mismo estado estacionario. Por lo tanto, las economías con niveles iniciales de capital tenderán a crecer más deprisa.

Esta hipótesis conocida como convergencia absoluta ha sido corroborada por Barro y Sala-i-Martin (1991) con estimaciones realizadas para los estados que conforman E.U.A., así como para los países de la OECD. Posteriormente, y como resultado de la crisis del modelo neoclásico, Barro y Sala-i-Martin (1992) desarrollaron el concepto de convergencia condicional, de esta forma se supone que economías con diferente estructura, puedan converger a pesar de la existencia de factores que condicionan la posición del estado estacionario de dichas economías. 
Las hipótesis de convergencia derivadas del modelo neoclásico permiten abordar parte de la problemática económica actual. Para el caso de México, recientemente se ha incrementado el interés por el estudio del crecimiento económico. Particularmente, las investigaciones se han enfocado al problema de la desigualdad regional como producto de un proceso de divergencia económica en los estados de la república.

El estudio del proceso de crecimiento económico de los estados ha corroborado la existencia de dos características: por un lado, la existencia de un proceso de convergencia, la cual disminuye la brecha de las diferencias en el bienestar entre los años 1980-1985 (Esquivel, 1999) y la reversión de este proceso entre los años 1985-1998 (Fuentes y Mendoza, 2001 y Arrollo, 2001), lo cual indica que se ha presentado un deterioro en la calidad de vida de la población en México.

Particularmente, Fuentes y Mendoza (2001) atribuyen a la inversión pública en infraestructura un papel relevante como freno de la desigualdad regional. Encuentran que en el periodo de convergencia 1980-1985, la infraestructura social representa un factor importante en la disminución de las diferencias regionales, no así en el caso de la infraestructura económica. En el periodo de divergencia 1985-1998, encuentran que las variables de infraestructura no son significativas, lo cual concuerda con el cambio en las funciones del Estado, que ahora incentiva más al elemento de inversión privada.

Cada uno de estos estudios incorpora implícitamente los factores que afectan el crecimiento de acuerdo a los años en que se realiza. Se considera importante analizar el proceso de convergencia en México en el periodo posterior a la crisis de 1994, lo cual recoge de manera implícita el efecto inercial de dicha crisis.

Aunado a lo anterior, se reconoce el papel que ha desempeñado la innovación tecnológica y el capital humano como factor del crecimiento económico. Mankiw, Romer y Weil (1992) incorporan el capital humano al modelo neoclásico para explicar el proceso de convergencia. Los modelos de crecimiento endógeno como alternativa teórica consideran la innovación tecnológica como un factor relevante del crecimiento (Romer, 1990 y Jones, 1999). Thwaities y Oakey (1985) consideran la innovación tecnológica regional como una actividad que fomenta el aumento de la producción regional.

Bajo esta perspectiva, se considera importante analizar la situación de la desigualdad económica regional en México a partir de 1994, resaltando el efecto que origina la innovación tecnológica regional. Así, en el análisis se considera a la variable de innovación tecnológica como condicionante de las diferencias en el estado estacionario de cada estado de la república.

\section{Estructura económica regional e innovación tecnológica}

Un análisis de la estructura del PIB per capita en el ámbito estatal permite observar el efecto de la crisis macrocconómica en la actividad económica regional. 
El impacto de corto plazo se caracterizó por un descenso en la producción per capita en todos los estados.

A partir de 1994, se inicia un proceso de recuperación de la actividad económica nacional, de tal suerte que la tasa de crecimiento media anual respecto al 2000 fue de $2.6 \%$. No obstante, no todos los estados lograron recuperar sus niveles iniciales, de actividad económica, lo cual generó una reestructuración de la producción per capita regional. En el cuadro siguiente se observa cómo entre los catorce estados con un mayor PIB per capita, algunos alcanzan posiciones más altas después de la mencionada reestructuración. Este es el caso de Nuevo León, Chihuahua, Coahuila y Sonora, que tuvieron tasas de crecimiento entre $3.2 \%$ y $4.3 \%$ en el periodo entre 1995 y 2000 . En tanto, sólo tres estados sufren un retroceso en su actividad económica per capita. Las tasas de crecimiento fluctuaron alrededor del $3 \%$.

Cuadro 1.

\begin{tabular}{|c|c|c|c|c|}
\hline \multicolumn{5}{|c|}{$\begin{array}{l}\text { Dinámica regional del PIB per capita en México 1995-2000 } \\
\text { Estructura porcentual y tasa de crecimiento media anual }\end{array}$} \\
\hline \multicolumn{2}{|c|}{1995} & \multicolumn{2}{|c|}{2000} & \multirow{2}{*}{$\frac{\text { TCMA95-00 }}{0.030}$} \\
\hline Distrito Federal & 8.18 & Distrito Federal & 8.36 & \\
\hline Campeche & 5.64 & Nuevo León & 5.80 & 0.036 \\
\hline Quintana Roo & 5.57 & Quintana Roo & 5.00 & 0.007 \\
\hline Nuevo León & 5.46 & Campeche & 4.96 & 0.004 \\
\hline Baja Californa Sur & 4.47 & Chihuahua & 4.69 & 0.043 \\
\hline Chihuahua & 4.25 & Coahuila & 4.56 & 0.039 \\
\hline Coahuila & 4.22 & Sollora & 4.14 & 0.032 \\
\hline Baja California & 4.11 & Baja California & 4.12 & 0.026 \\
\hline Sonora & 4.00 & Baja California Sur & 4.08 & 0.011 \\
\hline Querétaro & 3.61 & Querétaro & 4.05 & 0.046 \\
\hline Aguascalientes & 3.60 & Aguascalientes & 3.85 & 0.037 \\
\hline Colima & 3.49 & Tamlaulipas & 3.61 & 0.034 \\
\hline Tamaulipas & 3.44 & Colima & 3.42 & 0.022 \\
\hline Jalisco & 3.20 & Jalisco & 3.32 & 0.032 \\
\hline
\end{tabular}

Fuente: elaboración propia con datos del INEGI. EI PIB estatal es a precios de 1993. La población estatal se obtuvo del Censo de 2000 y del Conteo 95 resultados definitivos

Entre los estados con menor capital, el proceso de recuperación parece ser menos dinámico, entre ellos solamente Hidalgo, Michoacán y Puebla logran un ascenso notorio, con tasas de $3 \%$ similares al grupo con PIB per capita mayores. 
Cuadro 2.

\begin{tabular}{|c|c|c|c|c|}
\hline \multicolumn{4}{|c|}{$\begin{array}{c}\text { Dinámica regional del PIB per capita en México } \\
\text { Estructura porcentual y tasa de crecimiento media anual }\end{array}$} \\
\hline \multicolumn{2}{|c|}{1995} & \multicolumn{2}{c|}{2000} & TCMA95-00 \\
\hline Morelos & 2.91 & Durango & 2.96 & 0.036 \\
\hline Sinaloa & 2.86 & Morelos & 2.82 & 0.021 \\
\hline Durango & 2.80 & Edo. México & 2.62 & 0.028 \\
\hline Edo. México & 2.58 & Yucatán & 2.60 & 0.029 \\
\hline Yucatán & 2.55 & Sinaloa & 2.53 & 0.005 \\
\hline San Luis Potosí & 2.34 & San Luis Potosí & 2.39 & 0.029 \\
\hline Tabasco & 2.32 & Guanajuato & 2.29 & 0.024 \\
\hline Guanajuato & 2.32 & Puebla & 2.22 & 0.040 \\
\hline Veracruz & 2.14 & Hidalgo & 2.12 & 0.037 \\
\hline Nayarit & 2.07 & Michoacán & 2.03 & 0.035 \\
\hline Puebla & 2.04 & Nayarit & 2.01 & 0.021 \\
\hline Hidalgo & 1.99 & Veracruz & 2.00 & 0.014 \\
\hline Zacatecas & 1.97 & Tabasco & 1.96 & -0.002 \\
\hline Guerrero & 1.94 & Zacatecas & 1.87 & 0.017 \\
\hline Michoacán & 1.93 & Tlaxcala & 1.83 & 0.031 \\
\hline Tlaxcala & 1.77 & Guerrero & 1.74 & 0.007 \\
\hline Chiapas & 1.58 & Chiapas & 1.42 & 0.008 \\
\hline Oaxaca & 1.56 & Oaxaca & 1.42 & 0.010 \\
\hline
\end{tabular}

Fuente: elaboración propia con datos del INEGI. El PIB estatal a precios de 1993. La población estatal se obtuvo del Censo para el año 2000 y del Conteo de 1995 (resultados definitivos).

Lo anterior parece indicar que los estados con un mayor nivel del PIB per capita en 1995, mejoraron o permanecieron más o menos, en los mismos niveles de producción per capita. En contraparte, los estados más atrasados permanecieron en su posición o experimentaron un deterioro. De tal forma que, la evidencia empírica, sugiere la existencia de un aumento en la desigualdad económica regional y por lo tanto en el bienestar.

A continuación, se presenta una gráfica del logaritmo del PIB per capita respecto a su tasa de crecimiento entre 1995-2000. Se observa una relación positiva entre ambas variables, esto implica que los estados con un PIB per capita mayor en 1995 tendieron a crecer más rápido que los estados con niveles menores del PIB per capita en el mismo año. En este sentido, la gráfica muestra un proceso de divergencia entre los estados. 
Figura 1.

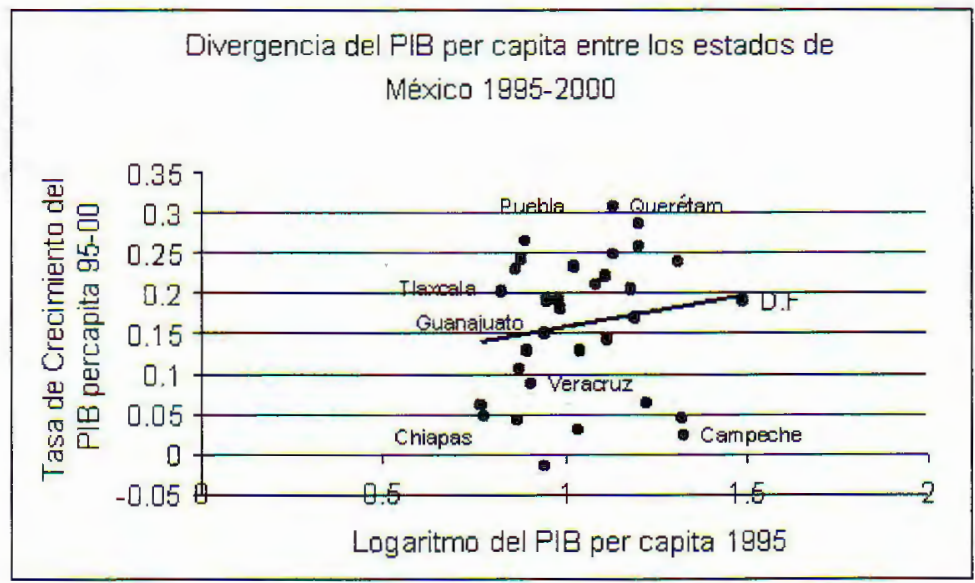

Con el fin de investigar los determinantes regionales que dan lugar al aumento en la desigualdad dentro del proceso de la dinámica económica regional, es importante considerar el papel que desempeña la innovación tecnológica en el proceso de divergencia económica regional.

En general, la innovación tecnológica en México muestra una disminución en su tasa de crecimiento a partir de 1993, acentuándose en 1995. La caída en la actividad de innovación experimentada en 1995 se extiende hasta el año 2000 , en el que se observa una recuperación en la producción de innovaciones tecnológicas. De hecho; la tasa de crecimiento media anual (TCMA) entre 1995 y 2000 fue de $0.9 \%$. El deterioro de la innovación tecnológica en 1995 refleja la sensibilidad de esta actividad ante el ambiente de crisis económica. Particularmente, esta disminución refleja las expectativas formadas acerca de los rendimientos futuros de la innovación y la reasignación de la inversión hacia actividades distintas.

En este contexto de deterioro y recuperación la actividad de innovación en México, se ha caracterizado por un proceso de reestructuración y convergencia relativa entre los estados. El análisis de la innovación tecnológica regional permite esclarecer el comportamiento general de la misma, así como también abordar el proceso de divergencia económica entre las regiones.

Cabe destacar que en el año de 1995, las solicitudes para proteger las innovaciones realizadas se encontraban distribuidas principalmente entre los estados que presentan un nivel más alto del producto interno bruto, es decir, en el Distrito Federal, Nuevo León y Jalisco. Entre los estados con menor número de patentes encontramos a Nayarit, Tlaxcala y Zacatecas, los cuales también presentan un bajo nivel de producto interno bruto. 
La recuperación de la innovación tecnológica en el 2000 hacia niveles similares de 1995, presenta una estructura regional diferente. Esto implica que la disminución de la producción de innovaciones en algunos estados fue compensada por otros. En 1995, el Estado de México tenía 5.7\% de la innovación nacional; en el 2000 aumentó a 9.8\%. El Distrito Federal en 1995 tenía un $45.8 \%$ disminuyendo su participación para el año 2000 a $40.7 \%$ (véase Cuadro $3)$.

En el proceso de reestructuración, catorce estados han presentado un aumento en sus niveles de innovación, siete permanecen en el mismo nivel y once han disminui 0 sus niveles de actividad de innovación. La estructura entre los diez estados con niveles más altos de innovación es estacionario, solamente Puebla se incorpora en el 2000. Entre los que conforman la estructura de 1995, seis tienen tasas de crecimiento positivas, que en su mayoría fluctúan entre $1 \%$ y $6 \%$, sólo el Estado de México y Querétaro presentan tasas de 11\% y $14 \%$ respectivamente (véase Cuadro 3 ).

Cuadro 3.

\begin{tabular}{|c|c|c|c|c|}
\hline \multicolumn{6}{|c|}{$\begin{array}{c}\text { Dinámica regional de la actividad de innovación tecnológica en México } \\
1995-2000 \text { Estructura porcentual y tasa de crecimiento media anual }\end{array}$} \\
\hline \multicolumn{2}{|c|}{1995} & \multicolumn{2}{c|}{2000} & TCMA95-00 \\
\hline Distrito Federal & 45.8 & Distrito Federal & 40.7 & -0.01 \\
\hline Nuevo León & 12.7 & Edo. México & 9.8 & 0.11 \\
\hline Jalisco & 7.8 & Nuevo León & 8.5 & -0.06 \\
\hline Edo. México & 5.7 & Jalisco & 7.4 & 0.00 \\
\hline Guanajuato & 3.3 & Querétaro & 5.4 & 0.14 \\
\hline Morelos & 2.6 & Pucbla & 3.6 & 0.15 \\
\hline Querétaro & 2.6 & Guanajuato & 3.4 & 0.01 \\
\hline Coahuila & 2.4 & Coahuila & 3.1 & 0.06 \\
\hline Baja California & 2.1 & Morelos & 3.1 & 0.04 \\
\hline Veracruz & 1.9 & Baja California & 2.7 & 0.05 \\
\hline Chihuahua & 1.7 & San Luis Potosí & 2.2 & 0.31 \\
\hline Puebla & 1.7 & Chihuahua & 2.0 & 0.04 \\
\hline Yucatán & 1.7 & Veracruz & 1.1 & -0.08 \\
\hline Tamaulipas & 1.4 & Tamaulipas & 0.9 & -0.07 \\
\hline Durango & 1.2 & Yucatán & 0.9 & -0.09 \\
\hline Aguascalientes & 0.9 & Aguascalientes & 0.7 & -0.05 \\
\hline Sinaloa & 0.9 & Oaxaca & 0.7 & 0.20 \\
\hline Tabasco & 0.7 & Sinaloa & 0.7 & -0.05 \\
\hline
\end{tabular}

Fuente: elaboración propia con información de CONACyT. Se emplea el número de patentes como medida aproximada de la innovación tecnológica 


\section{Cuadro 3}

(Continuación)

\begin{tabular}{|c|c|c|c|c|}
\hline \multicolumn{5}{|c|}{$\begin{array}{l}\text { Dinámica regional de la actividad de innovación tecnológica en México } \\
\text { 1995-2000 Estructura porcentual y tasa de crecimiento media annal }\end{array}$} \\
\hline \multicolumn{2}{|c|}{1995} & \multicolumn{2}{|c|}{2000} & TCMA95-00 \\
\hline Campeche & 0.5 & Sonora & 0.7 & 0.20 \\
\hline Chiapas & 0.5 & Michoacán & 0.4 & 0.00 \\
\hline Michoacán & 0.5 & Quintana Roo & 0.4 & 0.12 \\
\hline San Luis Potosí & 0.5 & Baja California Sur & 0.2 & - \\
\hline Colima & 0.2 & Campeche & 0.2 & -0.11 \\
\hline Hidalgo & 0.2 & Colima & 0.2 & 0.00 \\
\hline Oaxaca & 0.2 & Durango & 0.2 & -0.24 \\
\hline Quintana Roo & 0.2 & Guerrero & 0.2 & - \\
\hline Sollora & 0.2 & Hidalgo & 0.2 & 0.00 \\
\hline Baja California Sur & 0.0 & Tabasco & 0.2 & -0.17 \\
\hline Guerrero & 0.0 & Chiapas & 0.0 & -1.00 \\
\hline Nayarit & 0.0 & Nayarit & 0.0 & 0.0 \\
\hline Tlaxcala & 0.0 & Tlaxcala & 0.0 & 0.0 \\
\hline Zacatecas & 0.0 & Zacatecas & 0.0 & 0.0 \\
\hline Total & 100 & Total & 100 & 0.009 \\
\hline
\end{tabular}

Fuente: elaboración propia con información de CONACyT. Se emplea el número de patentes como medida aproximada de la innovación tecnológica

La estructura de los once estados con menor nivel de inmovación ha presentado un mayor cambio. De los estados que conforman la estructura de 1995, seis estados han experimentado un aumento cn los niveles de innovación. Colima, Hidalgo, Nayarit, Tlaxcala y Zacatecas han permanecido igual. Finalmente, estados como Campeche, Durango, Tabasco y Chiapas han presentado un descenso en su actividad de innovación.

Lo anterior, implica que los estados que inicialmente tenían un bajo nivel de innovación en 1995 han incrementado su esfuerzo tecnológico, con tasas de crecimiento que fluctúan entre el $12 \%$ y $31 \%$ como en el caso de San Luis Potosí. Esto ha permitido incrementar sus niveles de participación en el ámbito nacional, obscrvándose un proceso de convergencia en la producción de innovaciones.

El proceso de convergencia, al cual se incluyen Puebla y Chihuahua, no se manifiesta en todos los estados, ya que algunos hall mantenido el mismo nivel o disminuido la producción de innovaciones. Esto permite observar qué estados han contribuido aì descenso general de la actividad de innovación en México, resaltando el descenso del Distrito Federal quien tiene la mayor participación.

Las diferentes tasas de crecimiento de la innovación tecnológica logradas por cada estado y la concentración de la actividad de innovación, esclarecen la 
forma en que éstas influyen sobre el crecimiento económico en México. Por un lado, el volumen de innovaciones incrementa la probabilidad de que el efecto sobre el crecimiento económico general provenga de estados con mayor volumen de innovación. Por otro lado, el nivel de innovaciones de un estado en particular, puede ser suficiente para impulsar el crecimiento económico regional y crear externalidades positivas hacia el resto de las regiones a través de efectos de difusión.

La siguiente gráfica muestra el proceso de convergencia elı innovación. Algunos estados que en 1995 se encontraban atrasados en la actividad de innovación, presentan tasas de crecimiento más altas en la producción de inmovaciones tecnológicas en el 2002.

Figura 2.

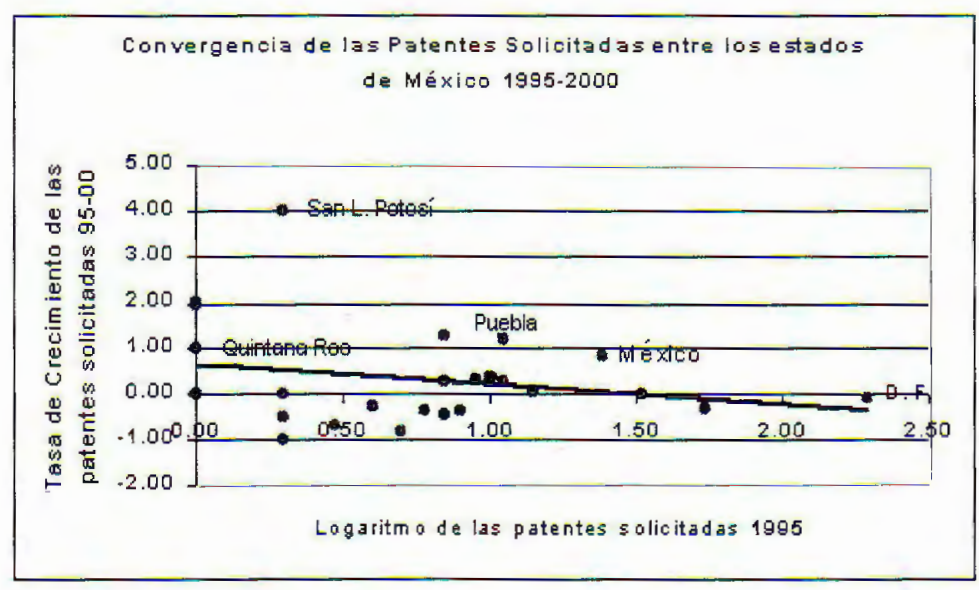

Es importante señalar que la coexistencia de un proceso de convergencia relativa en la actividad de innovación de los estados, con la disminución de las innovaciones, motiva en cierta forma un efecto de divergencia en el PIB per capita regional y por tanto en la desigualdad económica regional.

\section{Aspectos metodológicos}

A fin de corroborar el impacto de la innovación tecnológica en el proceso de divergencia económica de México, se desarrolla un modelo econométrico a partir del modelo de crecimiento usado para estudiar la hipótesis de convergencia absoluta:

$$
\gamma_{i t}=\alpha+(1-\beta) \log \left(\mathrm{y}_{i t-1}\right)+U_{i t},
$$

donde

$\gamma_{i t}=$ tasa de crecimiento del PIB per capita del estado $i$ entre el periodo $t$ 
$\mathrm{y} t-1$.

$\log \left(y_{i t-1}\right)==\operatorname{logaritmo}$ del PIB per capita para el estado $i$ en el periodo $t$.

La hipótesis es que existe convergencia entre los estados, por tanto se espera que el signo del coeficiente $(1-\beta)$ sea negativo. La estimación del impacto de la innovación tecnológica se realizará partiendo de la ecuación empírica estacionariocida por Barro, la cual es una extensión de la anterior, y se define de la siguiente forma:

$$
\gamma_{i t}=\alpha-\beta \log \left(\mathrm{y}_{i t-1}\right)+\phi X_{i t-1}+U_{i t},
$$

donde

$\gamma_{i t}=$ tasa de crecimiento del PIB per capita del estado $i$ entre el período $t$ y $t-1$.

$X_{i t-1}=$ vector de variables condicionantes del estado estacionario. En este caso, la innovación tecnológica en el estado $i$ en el período $t-1$.

El método que se utiliza es mínimos cuadrados ordinarios (MCO) para datos de sección cruzada.

\subsection{Base de datos}

La información estadística utilizada para la estimación del modelo considera el producto interno bruto a precios de 1993 y la población por estado en los años 1995 y 2000 , a partir de estas series de datos se construyó el producto interno bruto per capita a precios corrientes. La información se obtuvo del Instituto Nacional de Estadística, Geografía e Informática (INEGI).

Tanto en el ámbito internacional como en México, se considera como medida de innovación tecnológica el flujo de patentes solicitadas y concedidas a inventores nacionales y extranjeros residentes en nuestro país, así como a inventores nacionales que trabajan en el extranjero (OECD, 1997). La variable de innovación tecnológica es construida a partir del flujo de patentes solicitadas por cada uno de los estados de la República para los años 1995 y 2000, de tal forma que esta variable refleja el flujo esperado de innovaciones. La información de patentes fue recopilada a partir del sistema integral de información científica y tecnológica (SIICyT) del CONACyT.

Debe considerarse a las patentes como una medida imperfecta de la variable innovación, debido tanto a la práctica de secretos industriales ${ }^{1}$ como a las propensiones a patentes regionales. Por otro lado, esta medida refleja la actividad de innovación principalmente en las áreas siguientes: artículos de consumo, técnicas industriales, química y metalurgia, textil y papel, construcciones, mecánica, física y electricidad ${ }^{2}$.

1 El secreto industrial es utilizado para proteger una innovación patentable de la imitación. Sin embargo, la ley de propiedad industrial lo considera como un instrumento que protege una fase complementaria del proceso de innovación, que conlleva a una innovación patentable.

2 La clasificación de las patentes en México se realiza de acuerdo a la Clasificación Mundial de Patentes de la Organización Mundial de la Propiedad Industrial (OMPI). 
El número de patentes ha crecido recientemente en los países industrializados como instrumentos que permiten derechos monopólicos (OECD, 1997). Asimismo, estos países poseen los registros más completos y antiguos lo cual refleja su consolidación como instrumentos de protección tecnológica, permitiendo por otro lado el acceso a información y permiten realizar comparaciones entre países y regiones.

Entre los trabajos que han empleado las patentes como medida de innovación y cambio tecnológico podemos mencionar a: Hortsmann et al. (1985), quienes realizan un modelo de comportamiento de patentes para analizar la innovación en la firma; Stern et al. (2000), utilizan las patentes para estimar un modelo de capacidades tecnológicas; y por ultimo Griliches (1990) estudia las patentes en E.U.A. y su utilización en diversos estudios económicos. Este autor concluye que las patentes son indicadores objetivos que permanecen como única fuente para el estudio del cambio tecnológico.

\section{Divergencia económica e innovación tecnológica en México: evidencia empírica}

El marco analítico del modelo neoclásico permite abordar de manera formal el análisis del proceso de convergencia económica entre regiones. Para aplicar la hipótesis de convergencia absoluta, es posible utilizar la ecuación (1). Los resultados econométricos corroboran la hipótesis a priori en el apartado anterior, acerca de la existencia de un proceso de divergencia económica regional a partir de 1995. Esto es congruente con los resultados obtenidos en estudios realizados para periodos anteriores (véase Esquivel, 1999; Fuentes y Mendoza, 2001 y Arrollo, 2001).

El signo obtenido es positivo considerando la producción per capita de 1995 para cada estado. Este signo indica que los estados con un nivel más bajo crecieron menos de prisa que los estados con niveles iniciales más altos. Es decir, a partir de este año la desigualdad entre las regiones del país, medida en términos del PIB per capita, se incrementó. Esto conlleva a una distribución inequitativa del ingreso y a una polarización de los frutos del crecimiento. 


\begin{tabular}{c}
\hline Variable Dependiente: Tasa de Crecimiento del PIB per capita \\
Método: Mínimos Cuadrados Ordinarios
\end{tabular}

\begin{tabular}{|c|c|c|c|c|}
\hline Variable & Coefficient & Std. Error & t-Statistic & Prob. \\
\hline C & 0.079961 & 0.003198 & 25.00348 & 0.000 \\
LOG(PIB95) & 1.027637 & 0.001007 & 1020.278 & 0.000 \\
\hline
\end{tabular}

\begin{tabular}{|c|c|c|c|}
\hline \multicolumn{2}{|c|}{ Weighted Statistics } & \multicolumn{2}{c|}{ Unweighted Statistics } \\
\hline R-squared & 0.987267 & R-squared & 0.969574 \\
Adjusted R-sq & 0.987063 & Adjusted R-sq & 0.968560 \\
\hline
\end{tabular}

A partir de la ecuación (2) que recoge la hipótesis de convergencia condicional, se utiliza el siguiente modelo para estudiar el proceso de divergencia actual condicionado a la variable de innovación tecnológica:

$$
v_{i t}=\alpha+(1-\beta) \log \left(y_{i t-1}\right)+\log \left(\text { pats }_{i t-1}\right)+U_{i t} .
$$

Las variables son las mismas que en la ecuación (2), solamente se agrega la variable $\log \left(\right.$ pats $\left._{i t-1}\right)$, el logaritmo de las patentes del estado $i$ en el periodo $t-1$, que representa la innovación tecnológica.

Los resultados econométricos muestran que la variable innovación tecnológica es significativa en el proceso actual de divergencia económica regional. Particularmente, permanece el signo positivo de la variable $\log \left(y_{i t-1}\right)$ ante la inclusión de la variable innovación tecnológica. Esto corrobora el proceso de divergencia económica entre las regiones de México a partir de 1995.

La variable innovación tecnológica presenta siǵno positivo, esto indica el papel de la innovación en el proceso de divergencia. Los estados que tuvieron un nivel mayor de innovación tecnológica en 1995, son aquellos estados que tuvieron un crecimiento más rápido de la producción per capita. Este resultado, resalta no solamente el papel de la innovación tecnológica como factor de crecimiento económico. Además, implica el hecho de que aquellas regiones que realizaron una mayor actividad de innovación tecnológica son las regiones que tuvieron un crecimiento económico mayor. Esto es, la innovación tecnológica ha fomentado la divergencia económica.

Es necesario contextualizar la influencia de la actividad de innovación regional. El debilitamiento de dicha actividad se inserta no sólo en el marco de la crisis económica, sino que ha venido aparejado con un aumento de la actividad de innovación extranjera, como resultado de las reformas al sistema de propiedad industrial y apertura hacia los flujos tecnológicos a partir del tratado de libre comercio (TLC). Aboites (1999) menciona que como resultado de la reforma de la legislación en materia de propiedad industrial en el marco del TLC, 
las patentes solicitadas por extranjeros en México conformaron el $96 \%$ en el año 1996.

Así, en tanto los flujos de innovación extranjera tienen una respuesta positiva ante la recuperación económica posterior a 1995, y con la reducción de las restricciones de patentamiento, la innovación tecnológica nacional ha mostrado una tendencia decreciente a pesar de la recuperación económica experimentada en el año 2000.

Sin embargo, debemos decir que, la innovación tecnológica nacional se ha incrementado en la sección de químicos, disminuyendo en el resto de los secciones, a saber: eléctrico-electrónico, maquinaria no eléctrica, transportes y otras tecnologías. Aunque el sector de maquinaria no eléctrica perdió la primera posición, concentra junto con los químicos la mayoría de las innovaciones realizadas (Aboites, 1999).

Esto permite observar que la influencia de las innovaciones en la actividad económica regional depende del tipo y grado de integración con estos sectores, de la localización de los mismos en los estados e incluso de la difusión de información tecnológica entre regiones.

Variable Dependiente: Tasa de Crecimiento del PIB per capita Método: Mínimos Cuadrados Ordinarios

\begin{tabular}{|c|c|c|c|c|}
\hline Variable & Coefficient & Std. Error & t-Statistic & Prob. \\
\hline & & & & \\
C & 0.11331 & 0.021203 & 5.344102 & 0.000 \\
LOG(PIB95) & 1.005273 & 0.007702 & 130.5259 & 0.000 \\
PATS95 & 0.042027 & 0.002698 & 15.5782 & 0.000 \\
\hline
\end{tabular}

\begin{tabular}{|c|c|c|c|}
\hline \multicolumn{2}{|c|}{ Weighted Statistics } & \multicolumn{2}{|c|}{ Unweighted Statistics } \\
\hline R-squared & 0.989986 & R-squared & 0.970708 \\
Adjusted R-sq & 0.986985 & Adjusted R-sq & 0.968268 \\
\hline
\end{tabular}

Sin embargo, este hecho no oscurece el efecto positivo de la innovación en el crecimiento. Específicamente, ante las mayores tasas de crecimiento observadas en algunas regiones, como resultado de la innovación. Se desprende la necesidad de establecer políticas tecnológicas que promuevan la homogeneización de la actividad de innovación entre las diferentes regiones. Esto permitirá fomentar el crecimiento económico regional y ayudará a revertir el proceso actual de divergencia económica. 


\section{Conclusiones}

A partir de la hipótesis de convergencia absoluta derivada del modelo neoclásico, se corrobora la existencia de un proceso de divergencia económica entre las regiones de México, en el periodo 1995 a 2000. Este resultado es congruente con otros estudios realizados para periodos anteriores (1985-2000). Sin embargo, esto no implica que 1995 sea el inicio de la divergencia, sino que es en este año cuando el efecto implícito de la política económica implementada a principios de la década de los noventas comenzó a tener impacto.

La hipótesis de convergencia condicionada a la variable innovación tecnológica manifiesta, de nueva cuenta, la creciente desigualdad regional. La significancia estadística y el signo positivo de esta variable, no sólo resalta su influencia positiva en el crecimiento económico regional, sino también implica que los estados con mayor número de innovaciones tecnológicas han crecido más rápidamente.

En este sentido, la convergencia experimentada en la actividad de innovación entre los estados durante 1995 a 2000, no es suficiente para detener el proceso de divergencia económica regional. A ello contribuyen algunos factores que se desprenden de las características de la actividad de innovación en México. A saber, el grado de articulación de la actividad de innovación con la actividad económica regional, el grado de difusión de la tecnología entre regiones, la concentración de la actividad de innovación regional, la dotación regional de infraestructura tecnológica y capital humano. Incluso la influencia de la creciente competencia tecnológica, proveniente del flujo de innovación extranjera sobre la actividad de innovación y económica nacionales

Este resultado, lejos de disminuir la importancia de la actividad de innovación regional, permite vislumbrar una estrategia de política tecnológica regional que promueva la generación de innovación tecnológica más uniforme entre las regiones del país, con el fin de homogeneizar los niveles de innovación regional y contribuir a revertir el proceso de divergencia económica entre las regiones de México.

\section{Bibliografía}

Aboites A. J. y Manuel S. L. (1999). Innovación: Propiedad Intelectual y Estrategias Tecnológicas: la Experiencia de la Economía Mexicana. UAM-Xochimilco.

Arrollo, F. (2001). Dinámica del PIB de las Entidades Federativas de México: 1980-1999. Comercio Exterior, 51(7).

Barro, Robert y Xavier Sala-i-Martin (1991). Convergence Across States and Regions. Brooking Papers on Economic Activities, pp 107-182.

Barro, Robert y Xavier Sala-i-Martin (1992). Convergence. Journal of Political Economy 100(2), pp. 223-251.

Esquivel, G. (1999). Convergencia Regional en México, 1940-1995. El Trimestre Económico.

Fuentes, N. A. y J. E. Mendoza (2001). Infraestructura Pública y Convergencia Regional en México: 1980-1998. El Colegio de la Frontera Norte (Mimeo).

Griliches, Z. (1990). Patent Statistics as Economic Indicators: A Survey. National Bureau of Economic Research. Working Paper, 3001. 
INEGI (1997). Conteo de Población y Vivienda 1995: Resultados Definitivos. Perfiles Sociodemográficos, México.

Jones, I. C. (1999). Growth: with or without scale effects. American Economic Papers. Papers and Proceedings.

Mankiw, N. G., D. Romer y D. N. Weil (1992). A Contribution on The Empirics of Economic Growth. Quartely Journal of Economic. 107(2), pp. 407-437.

OCDE. (1997). Main Science and Technology Indicators. París, Clasificación Internacional de Patentes.

Romer, P. (1990). Endogenous Technological Change. (1990). National Bureau of Economic Research. Working Paper, 3210.

Sala-i-Martin, X. (1994). Apuntes de Crecimiento Económico. Antoni Bosch.

Stern S., M. E. Porter y J. L. Furman (2000). The Determinants of National Innovative Capacity. National Bureau of Economic Research. Working Paper, 7876.

Solow, R.(1956). A Contribution to The Theory of Economic Growth. Quartely Journal of Economic, 70(1), pp 65-94.

Thwaities, A. T. and R. P. Oakey . (1985) The Regional Economic Impact of Technological Change. St. Martins Press. New York. 